\title{
BMJ Open Scoping review study to identify how communities in the USA, Australia, New Zealand and Canada use quality improvement (QI) approaches to address community health and well- being
}

Tara Carr, ${ }^{1}$ Mallory Wolfe Turner (i) , ${ }^{2}$ Rohit Ramaswamy ${ }^{3}$

To cite: Carr T, Turner MW, Ramaswamy R. Scoping review study to identify how communities in the USA, Australia, New Zealand and Canada use quality improvement (Ql) approaches to address community health and well-being. BMJ Open 2019;9:e034302. doi:10.1136/ bmjopen-2019-034302

- Prepublication history for this paper is available online. To view these files, please visit the journal online (http://dx.doi. org/10.1136/bmjopen-2019034302).

Received 13 September 2019 Revised 22 October 2019 Accepted 19 November 2019

Check for updates

(c) Author(s) (or their employer(s)) 2019. Re-use permitted under CC BY-NC. No commercial re-use. See rights and permissions. Published by BMJ.

${ }^{1}$ Nutrition, University of North Carolina at Chapel Hill, Chapel Hill, North Carolina, USA

${ }^{2}$ Maternal and Child Health, University of North Carolina at Chapel Hill, Chapel Hill, North Carolina, USA

${ }^{3}$ Public Health Leadership and Maternal and Child Health,

UNC Gillings School of Global

Public Health, Chapel Hill, North Carolina, USA

Correspondence to

Mallory Wolfe Turner;

malwolfe@email.unc.edu

\section{ABSTRACT}

Introduction Both US and global communities lag on key health indicators. There has been limited progress in building capacity to improve health beyond the healthcare field. Yet, communities also need to engage in health improvement initiatives. A substantial body of literature describes standards and core components for quality improvement (QI) approaches in clinical settings. This study aims to determine how communities in the USA, Australia, New Zealand and Canada use QI approaches for health and well-being improvement and how such approaches compare to those in clinical settings.

Methods and analysis We developed a study protocol based on scoping review framework by Arksey and O'Malley, methodological advancements for scoping studies (Levac et a) and other published protocols. We developed research questions in an iterative process and used the Population, Intervention, Comparison, Outcomes strategy to determine eligibility criteria. Electronic databases deemed appropriate (Web of Science, Scopus, and Proquest Health Management) will be searched for studies that meet inclusion criteria. References of included studies will be included when relevant. Two reviewers will independently screen all abstracts and full-text studies for inclusion. A third reviewer will adjudicate disagreements that arise. An instrument will be developed to extract data from included studies. Quantitative and qualitative results will be reported.

Ethics and dissemination We developed this protocol to systematically conduct a scoping review of how US communities use $\mathrm{Ql}$ approaches to address community health and well-being. Results will benefit multiple stakeholders by informing how to better support, design and evaluate community well-being improvement interventions. Results will be distributed through peer-reviewed journals, conferences, presentations and a public health graduate course.

\section{INTRODUCTION}

\section{Background}

In our increasingly complex world, improving health and well-being for all has never been more important. WHO has defined health in terms of well-being for the past 70 years, ${ }^{1}$
Strengths and limitations of this study

While much is known about how clinical settings use quality improvement (QI) approaches, this scoping review will explore how QI approaches have been used to drive health and well-being improvements in community settings and how the approaches differ between these settings.

- Study results will inform a wide array of stakeholders including funders, researchers, practitioners and community members on how to better support, design and evaluate community well-being improvement interventions.

- No formal meta-analysis or analysis of the quality of the results will be done because the goal of this scoping review is to give an overview of what evidence exists and identify gaps.

- The consultation stage will not be performed, which limits stakeholder input on the other stages of the scoping review.

and the third Sustainable Development Goal (SDG) to ensure healthy lives and promote well-being for all has reinforced this idea. ${ }^{2}$ In the USA, a foundational principle of the Healthy People 2030 framework is that promoting the nation's health and well-being is a shared responsibility across national, state, tribal and community levels. ${ }^{3}$ Foundations such as the Robert Wood Johnson Foundation (RWJF) have adopted the creation of healthier communities as part of their action framework for creating a culture of health. ${ }^{4}$

Despite these major organisations' focus on community well-being, progress in building capacity to improve health beyond the healthcare field has been limited. Both US and global communities lag on key indicators of health. The 2017 Global Burden of Disease 
study reported that most countries will fail to meet the SDG targets of reducing deaths from non-communicable diseases. The chance of any country reducing overweight in children aged 2-4 years is $<5 \%$. $^{5}$ The US lags behind other wealthy countries in life expectancy, infant mortality and obesity. ${ }^{3}$ To address these issues, communities need to actively engage in health and well-being improvement initiatives and have the tools and capabilities to implement these improvement initiatives in a systematic way.

\section{Study rationale and conceptual framework}

This study explores the extent to which communities have used quality improvement (QI) methods to address wellbeing in their settings. We define a community as a group of people with diverse characteristics who are linked by social ties, share common perspectives and engage in joint action in geographical locations or settings. ${ }^{6}$ We adopt the definition by Batalden and Davidoff ${ }^{7}$ that QI is a systematic approach to improve outcomes and systems by building the capability of communities to identify, prioritise and develop solutions to local systems problems. There are several popular QI methods such as the Institute for Healthcare Improvement's Model for Improvement, Lean and Six Sigma, but in essence, each method focuses on mapping care delivery processes and systems, measuring the quality problem using data, identifying root causes for the problem, developing and implementing change strategies to address the problem and measuring the impact of the change. ${ }^{8}$ While the extent to which these methods can be causally attributed to improved outcomes in healthcare settings is still debated, ${ }^{9}$ there is a broad belief that if implemented rigorously, they can strengthen the system of care provision. ${ }^{1011}$

The extent to which community organisations or coalitions engaged in improving community well-being recognise or use these methods is unknown. The US Centres for Disease Control and Prevention's definition of community health improvement ${ }^{12}$ and a Community Health Improvement Process (CHIP) proposed by the Institute of Medicine (IOM) Committee on Using Performance Monitoring to Improve Community Health ${ }^{13}$ emphasise community needs assessments and the selection and implementation of health interventions. However, these definitions do not focus on the common elements of QI methods in healthcare such as process maps, outcome and process measurements or small tests of change that are instrumental in developing locally appropriate solutions for changing care delivery systems. ${ }^{14}$

While health systems are inherently similar because of the nature of healthcare delivery, we contend that the notion of community and extension community coalition structures is heavily dependent on the culture, economic markets and sociopolitical sphere of the countries in which they are found. Australia, New Zealand, Canada and the USA have relatively similar national contexts. These four nations are high-income countries that are part of the Anglosphere, have liberal market economies (which can be contrasted to continental Europe's more coordinated market economies), and experience health disparities between their White/Caucasian racial majority and their minority including indigenous populations. ${ }^{15}$

Communities are more diverse and complex than health systems. It stands to reason that community improvements are more likely to be adopted and sustained if QI methods used in healthcare settings can be used to develop locally tested community solutions or adapt evidence-based practices. ${ }^{16}$ This review aims to examine the extent to which QI methods are used in community health improvement work.

\section{METHODS AND ANALYSIS \\ Protocol design}

We selected a scoping review to answer our research questions because this method is best suited for identifying gaps in the research knowledge base, ${ }^{17}$ clarifying key concepts ${ }^{18}$ and reporting on what evidence exists rather than grading its quality. ${ }^{19}$

To design our scoping review protocol, we used scoping review framework by Arksey and O'Malley ${ }^{20}$; methodological advancements for scoping studies by Levac $e t a l^{21}$ and other published scoping reviews protocols. ${ }^{22-25}$ Our protocol consists of six stages: (1) identifying the research question; (2) identifying relevant studies; (3) selecting studies; (4) charting the data; (5) collating, summarising and reporting the results and (6) consulting with relevant stakeholders.

\section{Stage 1: identifying the research question}

We developed the research questions through an iterative process that involved identifying the research gap and refining our definitions. Research questions were informed by queries raised by graduate students during a QI course taught by one of the authors (RR). Students noted that hospital settings where most QI literature is set have well-defined and documented protocols, organisational structures with clearly identified roles and responsibilities, full-time staff paid to deliver service and buildings and infrastructure where care is provided. ${ }^{9} 26$ They questioned the applicability of these methods in community settings where coalition members may be loose affiliates who work part-time or volunteer, and no designated location where health improvement activities take place exists. This review seeks to answer three main questions:

1. How has community health improvement been defined?

2. What QI approaches have been used for community health improvement?

3. How are these approaches similar or different from those that have been implemented in the clinical setting (healthcare improvement)?

\section{Stage 2: identifying relevant studies}

\section{Databases}

The electronic databases of published literature identified as most appropriate for this study in consultation with a public health librarian are: Scopus, Web of Science and Proquest Health Management. These databases will be 


\section{Box 1 Search terms}

\section{Community and organisation search terms}

"Health department" OR (community AND Coalition*) OR (community And Partner*) OR Communit* OR (community AND Organization*) OR population*

AND

\section{Quality improvement search terms}

"Quality improvement" OR "improvement science" $\mathrm{OR}$ "continuous improvement" OR "improvement methods" OR "improvement tool" OR "quality initiative" $O R$ "quality tool" $O R$ "quality methods" $O R$ "driver diagram*" OR "value stream mapping" OR "model for improvement" OR "process improvement" OR (lean AND (quality OR management)) OR "six sigma" OR "lean six sigma" OR PDSA OR "plan do study act" OR "plan-do-study-act" OR "plan do check act" OR "plan-do-check-act" OR PDCA

\section{AND}

\section{Health and well-being search terms}

Health* OR WellbeingWell-being OR well-being OR "well being" OR Prevent* OR academic* OR education* OR justice OR "social determinant" $O R$ equit* $O R$ housing

\section{Exclude}

Genetic OR biopsy OR surgery OR "long-term care" OR "nursing homes" $O R$ perioperative $O R$ postoperative $O R$ radiology $O R$ "patient safety" OR "patient protection" OR "patient care" OR "patient discharge" OR "length of stay" OR "medical errors" OR "intensive care unit" OR "patient-reported outcomes" Or PROs OR "enhanced recovery pathways" OR ERPS OR "medical education" OR "postgraduate training" OR "clinical clerkship" OR "hospital administration" OR microbiome OR gene $O R$ engineering

searched to identify relevant studies, and bibliographies of included studies will also be searched and included when relevant.

\section{Search strategy}

Search terms were developed in collaboration with a public health librarian and include three primary categories of terms: community and organisation, QI and wellbeing (box 1). These terms will be searched under topic or abstract, title and keyword, depending on the database. We will limit our search to English language and to peer-reviewed studies indexed as taking place in the USA, Australia, New Zealand, Canada or 'undefined' country. We will include studies from the year 2000 or later because the use of systematic QI methods to improve health was limited prior to that time.

\section{Eligibility criteria}

We used the Population, Intervention, Comparison, Outcomes strategy to develop eligibility criteria for review. ${ }^{27}$ Inclusion and exclusion criteria and their operational definitions are listed in box 2 .

\section{Stage 3: study selection}

Study selection will occur in three phases. The first phase will finalise the search strings and the eligibility criteria. All three reviewers will independently review titles and abstracts for a $2 \%$ sample to identify studies as eligible
Box 2 Inclusion and exclusion criteria for acceptable papers and operational definitions

\section{Inclusion criteria}

Population or problem

- Well-being in community settings in the USA, Australia, New Zealand or Canada.

Intervention

- Any intervention addressing improvement of well-being using a quality improvement (QI) approach.

Comparison

- Any experimental or non-experimental study with or without comparison groups.

Outcomes

- Community-level well-being, or community capacity to improve.

\section{Exclusion criteria}

- Article focuses on drivers of improvement, effectiveness of improvement, etc, but does not use Ql approach or describe Ql process.

- Article describes approaches to improve community, coalition or programme function (eg, improve attendance of coalition members at meetings) without linkage to community well-being outcome.

- Intervention took place within the walls of a facility with no linkage to community setting.

\section{Operational definitions}

- Community. a group of people with diverse characteristics who are linked by social ties, share common perspectives and engage in joint action in geographical locations or settings.

- Community capacity. knowledge, motivation or skills to apply Ql approaches to community well-being.

- Community setting: where people eat, live, play, pray or participate in other voluntary activities, where attendance/participation is not mandatory.

- For example, school site (or any site of mandatory activity) if outside of mandatory attendance hours; outcome is measured at school-level but activities take place in community.

- Community well-being: any health (physical, mental), educational or social outcome measured at an aggregate level.

- Facility. school, correctional (juvenile, jail, prison), hospital, clinic, military.

- Intervention: an activity, evidence-based programme or policy that took place (ie, is not merely proposed).

- QI approach: any QI method, such as Lean, PDSA, Six Sigma or the Model for Improvement; or description of systematic process to improve community well-being.

or ineligible for full-text review using the inclusion and exclusion criteria. The reviewers will designate the studies as 'eligible', 'ineligible' or 'maybe' and aim for an interrater reliability (IRR) of $80 \%$ or higher. If this is not achieved, the definitions of the criteria will be reviewed and adjusted. Despite high IRR, if the search produces irrelevant studies, we will refine the search strings to narrow their focus.

In the second phase, two reviewers (TC and MWT) will each review half the titles and abstracts extracted using the search strings and the inclusion and exclusion criteria in box 2. As before, the reviewer will designate articles as 'eligible', 'ineligible' or 'maybe', with articles designated as 'maybe' moving on to the second stage. If articles do 


\section{Box 3 Elements of the data charting form}

1. Bibliographic information

- Study ID

- Article title

- Publication date

- Journal details (volume, issue, pages)

- Study location

- Extracted by

- Checked by

2. Researcher details

- Authors and affiliations (as presented on paper)

3. Scoping review inclusion and exclusion criteria

- Study population details (age, sex, race/ethnicity, sample size)

- Community setting

- Concept

- Context

- Type of study

4. Aims and methods

- Study aims/objectives

- Methodology

- Quality improvement approach

- Intervention details (type, activity, duration, components)

5. Study outcomes

- Classification of community health or well-being outcomes (physical, mental, educational, social well-being or community capacity to improve)

- Description of study outcomes and results

6. Emergent categories

- Any further information that emerges as important to the study not captured above, and agreed on by the study team

not have an abstract and are not obviously excludable based on title, they will be designated as 'maybe'. Third, the two reviewers will both review the full text of each article designated 'eligible' or 'maybe' that met the inclusion criteria to determine if it will be included in the scoping review. If conflict arises, a third reviewer (RR) will read the full text and consult with TC and MWT to make a final decision.

\section{Stage 4: data collection and charting the results}

Using the JBI Reviewer's Manual scoping review extraction template ${ }^{28}$ and the Preferred Reporting Items for Systematic Reviews and Meta-Analyses Extension for Scoping Reviews (PRISMA-ScR): Checklist and Explanations guides, ${ }^{29}$ we will develop an initial draft of a standardised data charting instrument to extract relevant study information. Elements of the draft data charting form are listed in box 3 .

Data to be extracted from the included studies will comprise standard information, such as author, year of publication and study objectives. We will also extract information to examine the application of QI methods in community health interventions, intervention details (type, activity, duration, components), study population and sample size, study setting and a description of outcome measures. If the need for novel data extraction categories emerges during the data collection process, or we find eligible abstracts with missing data, consultation within our research team will guide decisions and be reported with the findings.

\section{Stage 5: data summary, synthesis and report of the results}

We will provide both a qualitative and quantitative summary of the scoping review results. PRISMA guidelines $^{30}$ will guide our qualitative, thematic analysis of the extracted data. We will report on a priori themes related to our research questions and any emergent themes that arise. The goal of the scoping review is to give an overview of what exists and identify gaps, not to metaanalyse or analyse the quality of the studies. The quantitative summary will detail the number and type of studies included.

Assessing study quality is optional in scoping reviews because one of the objectives of conducting such a review is to improve the precision of research questions based on the literature rather than to assess the quality of the published evidence to answer a specific research question. As stated by Munn et al, some of the objectives of scoping reviews are to identify the types of evidence available in a field, to clarify definitions and concepts and to identify knowledge gaps. ${ }^{31}$ This is the context in which our review is performed. There is no clear definition of what community health improvement means or how QI methods have been applied to these settings, and it is possible that our review will uncover a number of heterogeneous approaches that are difficult to compare. Our emphasis in this review will therefore be on describing the kinds of studies that present the use of QI methods in communities with the goal of providing guidance on identifying the kinds of studies that might need to be conducted before a systematic review is appropriate.

\section{Stage 6: consultation with stakeholders}

We will not perform consultation for the present scoping review. While community health interventions using QI approaches may exist in the practice setting, reporting and dissemination may not be abundant in the grey literature. Moreover, engaging stakeholder consultation in the process would involve developing a credible protocol and identifying a diverse group of community members and subject matter experts for consultation. We may complete this stage separately at a later date.

\section{Patient and public involvement}

No patient was involved.

\section{ETHICS AND DISSEMINATION}

This study protocol has been developed to systematically conduct a scoping review of English-language, peerreviewed articles published since 2000 and synthesise data to identify how communities in the USA, Australia, New Zealand and Canada use QI approaches to address community health and well-being. While this study will neither grade evidence nor draw relationships between use of QI approaches and community-level well-being 
outcomes, results will provide a better understanding of: (1) how community health improvement has been defined and conceptualised; (2) how and to what extent QI approaches are being used in the community setting to address health and (3) the similarities or differences of how such approaches manifest in the community versus clinical setting. Evidence from recently published studies, such as those on the Spreading Community Accelerators through Learning and Evaluation initiative funded by the RWJF, ${ }^{32-34}$ indicates that this novel body of inquiry is of current interest and is being explored. Study results will inform funders, researchers, practitioners and community members on how to better support, design and evaluate community health and well-being improvement interventions. Dissemination of study results will occur through peer-reviewed journals, conferences, presentations and a public health graduate course.

Contributors RR conceptualised the idea and contributed to writing and editing the protocol. TC and MWT helped refine the idea and develop and write the protocol.

Funding The authors have not declared a specific grant for this research from any funding agency in the public, commercial or not-for-profit sectors.

Competing interests None declared.

Patient consent for publication Not required.

Ethics approval Ethics approval through the Institutional Review Board was not obtained because this study does not involve human subjects.

Provenance and peer review Not commissioned; externally peer reviewed.

Open access This is an open access article distributed in accordance with the Creative Commons Attribution Non Commercial (CC BY-NC 4.0) license, which permits others to distribute, remix, adapt, build upon this work non-commercially, and license their derivative works on different terms, provided the original work is properly cited, appropriate credit is given, any changes made indicated, and the use is non-commercial. See: http://creativecommons.org/licenses/by-nc/4.0/.

\section{ORCID iD}

Mallory Wolfe Turner http://orcid.org/0000-0002-6807-7894

\section{REFERENCES}

1 World Health Organization. Constitution, 2019. Available: https:// www.who.int/about/who-we-are/constitution [Accessed 2 Jun 2019].

2 United Nations. Goal 3: ensure healthy lives and promote well-being for all at all ages. sustainable development goals. Available: https:// www.un.org/sustainabledevelopment/health/ [Accessed 3 Jun 2019].

3 Office of Disease Prevention and Health Promotion. Healthy people 2030 framework, 2019. Available: healthypeople.gov. https://www. healthypeople.gov/2020/About-Healthy-People/DevelopmentHealthy-People-2030/Framework [Accessed 3 Jun 2019].

4 Robert Wood Johnson Foundation. Building a culture of health, 2018. Available: https://www.rwjf.org/en/how-we-work/building-a-cultureof-health.html [Accessed 3 Jun 2019].

5 Lozano R, Fullman N, Abate D, et al. Measuring progress from 1990 to 2017 and projecting attainment to 2030 of the health-related sustainable development goals for 195 countries and territories: a systematic analysis for the global burden of disease study 2017. Lancet 2018;392:2091-138.

6 MacQueen KM, McLellan E, Metzger DS, et al. What is community? an evidence-based definition for participatory public health. $\mathrm{Am} \mathrm{J}$ Public Health 2001;91:1929-38.

7 Batalden PB, Davidoff F. What is "quality improvement" and how can it transform healthcare? Qual Saf Health Care 2007;16:2-3.

8 The Health Foundation. Quality improvement made simple. London: The Health Foundation, 2013.

9 Dixon-Woods M, Martin GP. Does quality improvement improve quality? Future Hosp J 2016;3:191-4.
10 Ramaswamy R, Reed J, Livesley N, et al. Unpacking the black box of improvement. Int J Qual Health Care 2018;30:15-19.

11 Boyd S, Aggarwal I, Davey P, et al. Peripheral intravenous catheters: the road to quality improvement and safer patient care. $J$ Hosp Infect 2011;77:37-41.

12 Centers for Disease Control and Prevention. CDC community health improvement navigator, 2015. Available: https://www.cdc.gov/ chinav/index.html [Accessed 3 Jun 2019].

13 Institute of Medicine [IOM]Durch JS, Bailey LA, Stoto MA, eds. Improving Health in the Community: A Role for Performance Monitoring. (Durch JS. Washington (DC): National Academies Press (US), 1997.

14 Berwick DM. A primer on leading the improvement of systems. BMJ 1996;312:619-22.

15 Pulver LJ, Haswell MR, Ring I. Indigenous Health - Australia, Canada, Aotearoa New Zealand and the United States - Laying Claim to a Future That Embraces Health for Us All. World Health Organization, 2010. https://www.who.int/healthsystems/topics/financing/ healthreport/IHNo33.pdf

16 Mondoux S, Shojania KG. Evidence-based medicine: a cornerstone for clinical care but not for quality improvement. J Eval Clin Pract 2019;25:363-8.

17 Crilly T, Jashapara A, Ferlie E. Research utilisation \& knowledge mobilisation: a scoping review of the literature 2010.

18 de Chavez AC, Backett-Milburn K, Parry O, et al. Understanding and researching wellbeing: its usage in different disciplines and potential for health research and health promotion. Health Educ $J$ 2005;64:70-87.

19 DeCaria JE, Sharp C, Petrella RJ. Scoping review report: obesity in older adults. Int J Obes 2012;36:1141-50.

20 Arksey H, O'Malley L. Scoping studies: towards a methodological framework. Int J Soc Res Methodol 2005;8:19-32.

21 Levac D, Colquhoun H, O'Brien KK. Scoping studies: advancing the methodology. Implementation Sci 2010;5.

22 Jolley RJ, Lorenzetti DL, Manalili K, et al. Protocol for a scoping review study to identify and classify patient-centred quality indicators. BMJ Open 2017;7:e013632.

23 Halas G, Schultz ASH, Rothney J, et al. A scoping review protocol to map the research foci trends in tobacco control over the last decade. BMJ Open 2015;5:e006643.

24 Archibald D, Patterson R, Haraldsdottir E, et al. Mapping the progress and impacts of public health approaches to palliative care: a scoping review protocol. BMJ Open 2016;6: 012058.

25 Schoeller SD, Lima DKS, Martins MM, et al. Protocol for a scoping review on nursing care and the autonomy of disabled persons. BMJ Open 2018;8:e022106.

26 Taylor MJ, McNicholas C, Nicolay C, et al. Systematic review of the application of the plan-do-study-act method to improve quality in healthcare. BMJ Qual Saf 2014;23:290-8.

27 Liberati A, Altman DG, Tetzlaff J, et al. The PRISMA statement for reporting systematic reviews and meta-analyses of studies that evaluate health care interventions: explanation and elaboration. PLOS Med 2009;6:e1000100.

28 JBI Reviewer's Manual - JBI GLOBAL WIKI. Appendix 11.1 JBI template study details, characteristics and results extraction instrument. Available: https://wiki.joannabriggs.org/display/ MANUAL/Appendix+11.1+JBI+template+study+details\%2C+ characteristics+and+results+extraction+instrument [Accessed 8 Jun 2019].

29 Tricco AC, Lillie E, Zarin W, et al. PRISMA extension for scoping reviews (PRISMA-ScR): checklist and explanation. Ann Intern Med 2018;169:467-73.

30 Moher D, Liberati A, Tetzlaff J, et al. Preferred reporting items for systematic reviews and meta-analyses: the PRISMA statement. PLOS Med 2009;6:e1000097.

31 Munn Z, Peters MDJ, Stern C, et al. Systematic review or scoping review? guidance for authors when choosing between a systematic or scoping review approach. BMC Med Res Methodol 2018;18:143

32 Scaccia J. Community health improvement and the community psychology competencies. Glob J Community Psychol Pract 2017;8.

33 Reid A, Abraczinskas M, Scott V, et al. Using collaborative coalition processes to advance community health, well-being, and equity: a multiple-case study analysis from a national community transformation initiative. Health Educ Behav 2019;1090198119838833.

34 Domlyn AM, Wandersman A. Community coalition readiness for implementing something new: using a Delphi methodology. $J$ Community Psychol 2019;47:882-97. 